\title{
Correlated transitions in symmetric and dominant fission modes, and multipole moments of fission fragments
}

\author{
Satoshi Chibal, Mark D. Usang ${ }^{1,2}$, Chikako Ishizuka ${ }^{l}$ Fedir Ivanyuk ${ }^{1,3}$ and Zhang Xuan ${ }^{l}$ \\ ${ }^{1}$ Tokyo Institute of Technology, 152-8550, Tokyo, Japan \\ ${ }^{2}$ Malaysia Nuclear Agency, 43000, Bangi, Malaysia \\ ${ }^{3}$ Institute of Nuclear Research, 03028, Kiev, Ukraine
}

\begin{abstract}
Systematic and anomalous trends in fragment mass and TKE (total kinetic energy) distributions are investigated in terms of 4D Langevin model developed at Tokyo Tech. We have found that correlated transitions in symmetric components and dominant modes (symmetric v.s. asymmetric) can explain the prominent systematic and anomalous features of fission observables. We have also elucidated that interplay between spherical and deformed magicity at $\mathrm{A}=132$ and $\mathrm{A}=142$ to 144 is important in both observables.
\end{abstract}

\section{Introduction}

Fission is the most important and fundamental physics process in nuclear applications, but it is important also in r-process nucleosynthesis in binary neutron-star merger as fission recycling is now believed to be taking place. Recently, it is strongly recognized that signals from fission (or fission products) plays essential role in nuclear security and safeguards. Thanks to recent progress in experimental technique, nuclear theory and computer science, our understanding on nuclear fission is in a stage of rapid progress, although it is still a mysterious phenomenon due to its nature as a large-amplitude collective motion of many nucleon systems.

Recently, we have carried out a systematic effort to construct a computational framework of nuclear fission, starting from compound nuclei through scission to emission prompt neutrons till $\beta$-decay, with less number of parameters and/or hypotheses. Now, our main interest is to connect the outcome of 4-D Langevin approach[1] to prompt neutron emission to predict multiplicities of prompt neutrons as a function of mass number of fission fragments, the so-called saw-tooth structure.

In this work we wish to describe our recent work with the 4D Langevin model developed at Tokyo Institute of Technology. Here, it will be explained how the systematic and anomalous trends in the fragment mass and TKE distributions can be explained as a correlated transition of symmetric mode from super-long to super short, and the dominant mode lying at the asymmetric mode, shifting to symmetric, and then asymmetric modes again as $\mathrm{Z}^{2} / \mathrm{A}^{1 / 3}$ of the fissioning nuclei increases. Then, we calculated $\angle \mathrm{Q}_{20}>$ and $\angle \mathrm{Q}_{30}>$ of fission fragments, and obtained a direct evidence of the super-long mode for the symmetric components in the fission of actinide region, and large $\left\langle\mathrm{Q}_{30}>\right.$ for mass region of 142-144 corresponding to softness to the octupole deformation, which is traditionally interpreted as originated from deformed shell. 


\section{Method}

We describe the fission process within the Langevin approach [2], i.e., by solving the equations for the time evolution of the shape of nuclear surface of fssioning system. Here, we implicitly assume that a fissioning nucleus can be divided into 2 systems in thermal equilibrium at temperature $T$, namely, 1 ) heat bath consisting of nucleons and 2) the system of our interest, which consists of collective shape coordinates which are chosen to describe the change of nuclear shape during fission in a convenient way. These 2 systems are assumed to exchange energy via friction and random force. For the shape parametrization we use that of two-center model (TCM)[3] with 4 deformation parameters $\mathrm{q}_{\mu}=\left\{\mathrm{z}_{0} / \mathrm{R}_{0}, \delta_{1}, \delta_{2}, \alpha\right\}$, where $\mathrm{z}_{0} / \mathrm{R}_{0}$ refers to the distance between the centers of left and right Nilsson-type oscillator potentials with $\mathrm{R}_{0}=1.2 \mathrm{~A}^{1 / 3}, \mathrm{R}_{0}$ being the radius of spherical nucleus with the mass number $\mathrm{A}$. The parameters $\delta_{i}$, where $i=1,2$, describe the deformation of the right and left fragment tips. The fourth parameter $\alpha$ is the mass asymmetry and the fifth parameter of TCM shape parametrization $\varepsilon$ was kept constant, $\varepsilon=0.35$, in all our calculations. This value of $\varepsilon$ has been decided in a detailed analysis for fission of $n+{ }^{235} \mathrm{U}$ system.

The first order stochastic differential equations (Langevin equations) for the time dependence of collective variables $q_{\mu}$ and the conjugated momenta $p_{\mu}$ are given as follows:

$$
\begin{aligned}
& \frac{d q_{\mu}}{d t}=\left(m^{-1}\right)_{\mu v} p_{v}, \\
& \frac{d p_{\mu}}{d t}=\frac{\partial F(q, T)}{\partial q_{\mu}}-\frac{1}{2} \frac{\partial m_{\mu}^{-1}}{\partial q_{\mu}} p_{v} p_{\sigma}-{ }_{\mu} \gamma_{\nu} m_{v \sigma}^{-1} p_{\sigma}+{ }_{\mu}^{\sigma} g_{v} R_{v}(t),
\end{aligned}
$$

where the sums over the repeated indices are assumed. In Eqs. (1) and (2), the $F(q, T)$ is the temperature dependent free energy of the system, and $\gamma_{\mu \nu}$ and $\left(\mathrm{m}^{-1}\right)_{\mu \nu}$ are the friction and inverse of mass tensors, $\mathrm{g}_{\mu \nu}$ is the strength of random force and $R_{\nu}$.denotes a random force term corresponding to white noise.

The free energy $F(q, T)$ is calculated as the sum of liquid drop deformation energy and the temperature dependent shell correction $\delta F(q, T)$. The damping of shell correction $\delta F(q, T)$ with the excitation energy is described in detail in [4]. The single particle energies are calculated with the 2-center deformed Woods-Saxon potential $[5,6]$ fitted to the aforementioned TCM shape parameterizations. It is to be noted the free energy is equal to potential energy at zero temperature. The main part of the shell correction consists of Strutinsky correction and BCS paring correction to the free energy.

The collective inertia tensor $m_{\mu v}$ is calculated within the Werner-Wheeler approximation [7] and for the friction tensor $\gamma_{\mu \nu}$ we used the wall-and-window formula[7,8]. The factors $g_{\mu v}$ are related to the temperature and friction tensor via the modified Einstein relation ,

$$
g_{\mu \sigma} g_{\sigma \nu \mu \nu}=T^{*} \gamma \text {, with } T^{*}=\frac{\hbar \omega}{2} \operatorname{coth} \frac{\hbar \omega}{2 T} \text {, }
$$

where $T^{*}$ is the effective temperature[9]. The parameter $\omega$ is the local frequency of collective motion [10]. The minimum of $T^{*}$ is given by $\hbar \omega / 2$. The temperature $T$ in this context is related to the initial energy $E^{*}{ }_{(i n)}$ and the excitation energy $E^{*}$ by, 


$$
E^{*}=E_{g s}+E_{(i n)}^{*}-\frac{1}{2} m_{\mu \nu t, v}^{-1} p-V_{p o t}(q, T \quad 0) a \mp^{2},
$$

where $\mathrm{V}_{\text {pot }}$ is the potential energy and $a$ is the level density parameter. More details are given in our earlier publications [11-12].

Initially, the momenta $p_{\mu}$ are set to zero, and calculations are started from the ground state deformation. Such calculations are continued until the trajectories reach the "scission point", which was defined as the point in deformation space where the neck radius turns into zero

\section{Results and discussion}

Calculated fragment mass and TKE correlations for a set of selected actinide nuclei are shown in Fig. 1.

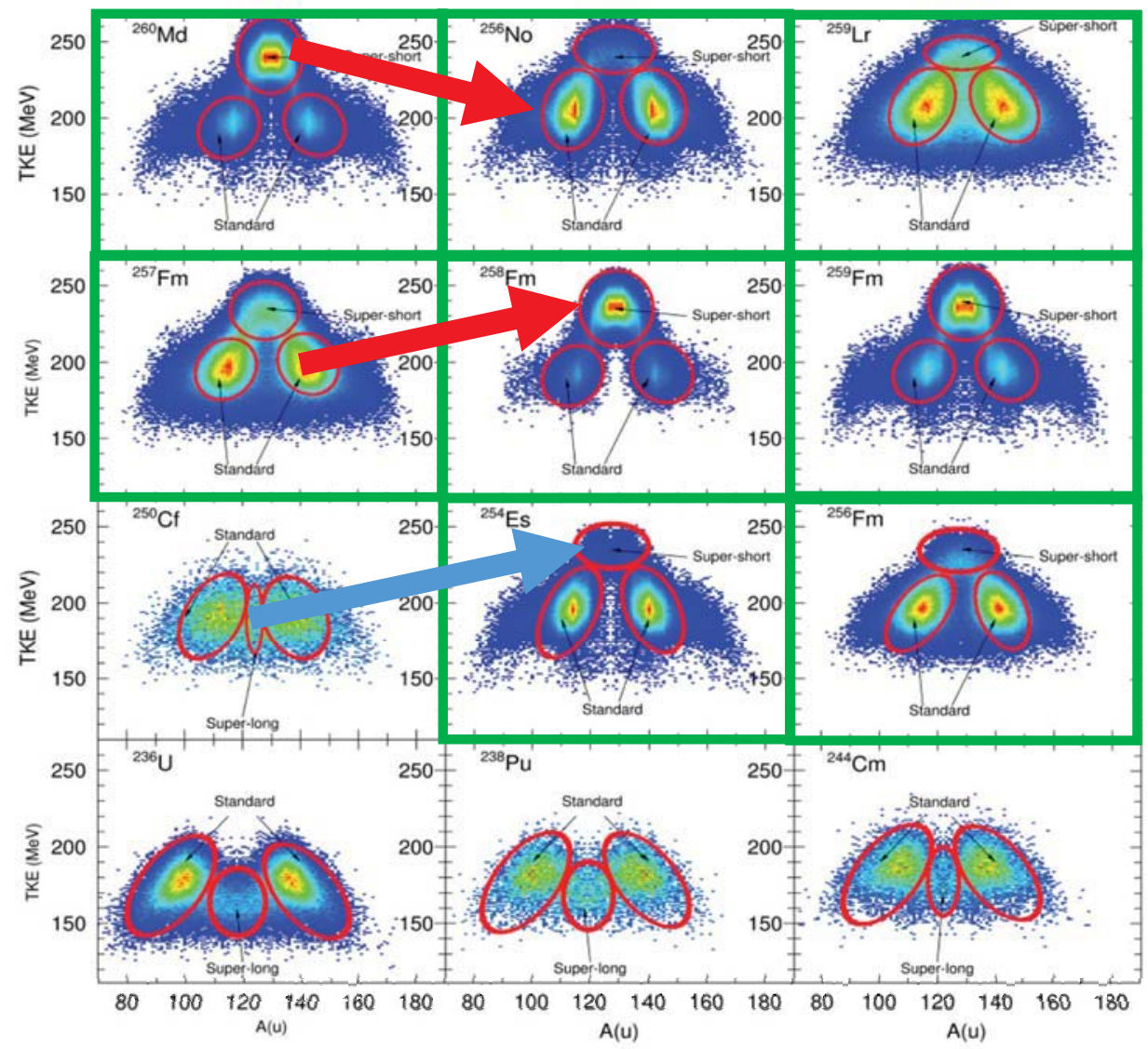

Fig. 1 Fragment mass and TKE correlations calculated by 4D Langevin model. The asymmetric (standard mode) and symmetric components are enclosed by red circles. The blue arrow shows a point of transition of the symmetric component from superlong to supershort mode, and the red arrows show transitions of the dominant mode from the standard to supershort mode and a reverse direction.

We can clearly observe a transition of the symmetric component, going from the superlong to supershort modes between ${ }^{250} \mathrm{Cf}$ and ${ }^{254} \mathrm{Es}$, and the symmetric component stays 
at the supershort mode for heavier systems. On the contrary, the dominant mode makes a transition in between ${ }^{257} \mathrm{Fm}$ to ${ }^{258} \mathrm{Fm}$ from asymmetric to symmetric mode, (mass distribution changes from 2-peaked structure to a single-peaked one) while it transits back to the asymmetric mode in between ${ }^{260} \mathrm{Md}$ to ${ }^{256} \mathrm{No}$ (transition of mass distribution from single-peak to 2-peaked distribution).

We can calculate the average TKE of the symmetric and asymmetric components separately, and they are plotted as a function of $\mathrm{Z}^{2} / \mathrm{A}^{1 / 3}$ of the fissioning nucleus in Fig. 2 . It can be seen from this figure that the average TKE for the asymmetric component, "Calc. TKEasy", increases monotonically, and it is in very good agreement with systematics proposed by Unik [13] and Viola [14]. On the other hand, the average TKE for the symmetric component makes a sudden jump at $\mathrm{Cf}$ to $\mathrm{Fm}$ as indicated by the blue arrow. Therefore, there is a clear transition of the fission mechanisms here. The black dots in Fig. 2 denote position of the dominant mode. It is on the standard mode for nuclei having smaller $\mathrm{Z}^{2} / \mathrm{A}^{1 / 3}$, showing that the mass distribution has 2 peaks (since it is in the asymmetric mode), then makes a sudden jump to the symmetric component in the middle of the Fm isotopes so the mass distribution turns into a single-peaked structure, then suddenly transits back to the asymmetric mode as the red arrows indicated. By the combination of these 2 transitions, we can explain very well the sudden change of the mass distribution from ${ }^{257} \mathrm{Fm}$ to ${ }^{258} \mathrm{Fm}$, and systematics and anomaly in the average TKE of fission fragments.

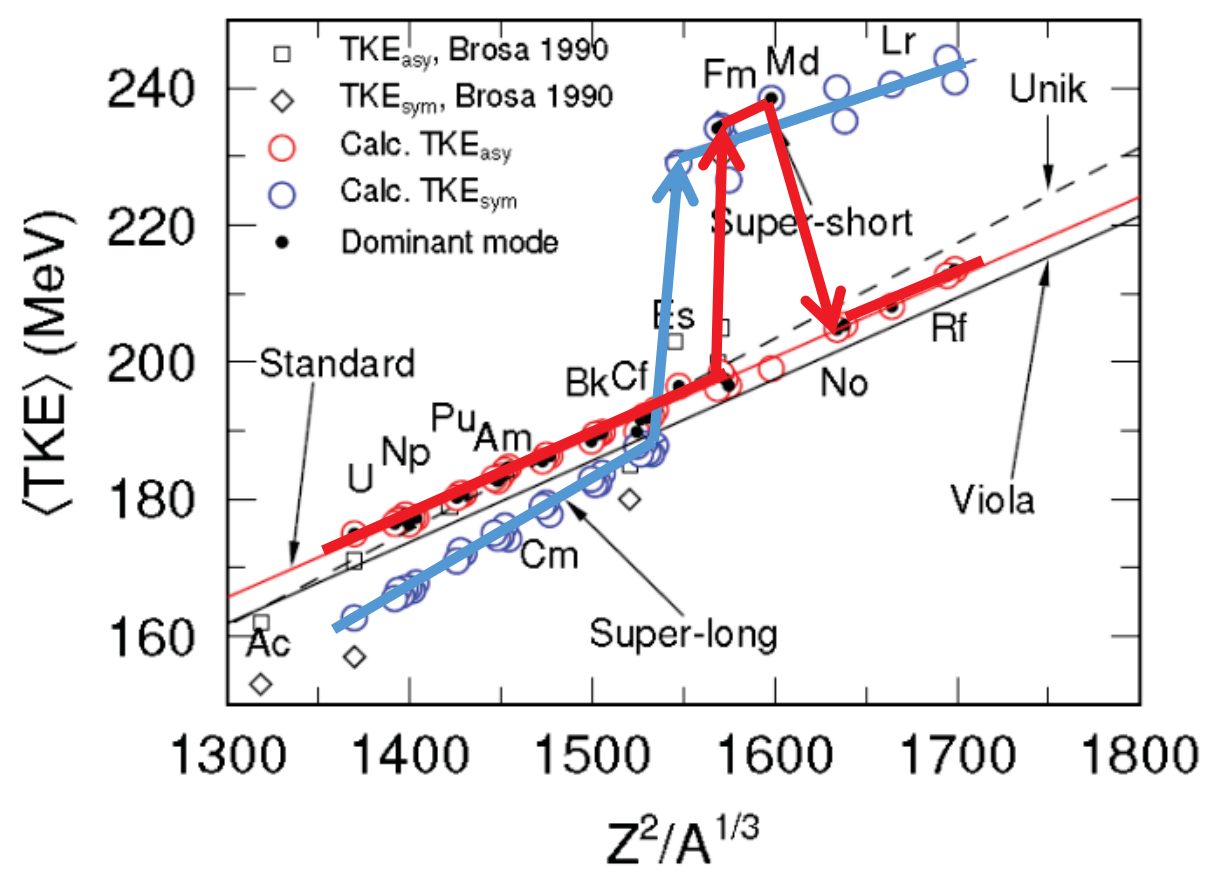

Fig. 2 Average total kinetic energy $(<\mathrm{TKE}>)$ of the symmetric and asymmetric components as a function of $\mathrm{Z}^{2} / \mathrm{A}^{1 / 3}$ of the fissioning nuclei. The calculated values are

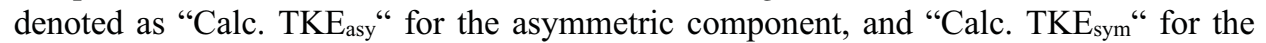
symmetric component. The dominant mode is indicated by the black dots. The square and diamond symbols are experimental data reported in Brosa's paper 15].

Figure 3 shows mass distributions (upper panels) and mass-quadrupole moment (bottom panels) for ${ }^{256} \mathrm{Fm}$ and ${ }^{258} \mathrm{Fm}$. It is well known that the mass distribution becomes from 2 peaked structure typical for actinides to a sharply single-peaked structure here. We 
think we know the reason. If we see the quadrupole moment of ${ }^{258} \mathrm{Fm}$, the average value at each fragment mass number, shown by the black histogram, touches down to $\mathrm{Q}_{20}=0$ at $A=132$. It clearly shows that the fission mechanisms is dominated by the spherical magicity of ${ }^{132} \mathrm{Sn}$, and, since the mass number is close enough to twice 132 , the compliment fragment should have a very similar mass number, so both the light and heavy fragments form a common single peak. On the contrary, the average $\mathrm{Q}_{20}$ dese not touches down to 0 for ${ }^{256} \mathrm{Fm}$, but stays finite. It indicates that the fission is affected by the deformed magicity around $A=142-144[16]$. Since the mass number of the heavy fragments is large enough, the compliment light fragments have mass numbers which are sufficiently small to form another peak, giving rise to the 2-peaked structure. We carried out similar analysis for $\mathrm{Q}_{30}$, but we will omit discussion on it here.

\section{Conclusions}

We have investigated the mass-TKE correlation of fission fragments by 4D Langevin model. The calculated data were decomposed into symmetric and asymmetric components and we have seen how they behave when fissioning system changes. We have observed that there is a clear transition of 1) the symmetric component from the superlong to supershort mode, and 2) dominant mode from asymmetric to symmetric, then to asymmetric component. We have shown that combination of these 2 transitions can account for the systematic and anomalous trends in mass and TKE distributions in the region of actinides. Furthermore, the mass-quadrupole deformation can explain why the sudden transition from the 2-peak structure to a single-peak structure in mass distribution between ${ }^{256} \mathrm{Fm}$ (actually ${ }^{257} \mathrm{Fm}$ ) and ${ }^{258} \mathrm{Fm}$; fission in nuclei lighter than ${ }^{257} \mathrm{Fm}$ is affected by deformed magicity at $A=142-144$ as suggested by Scamps and Simenel, while that of ${ }^{258} \mathrm{Fm}$ is strongly affected by spherical magicity of ${ }^{132} \mathrm{Sn}$.
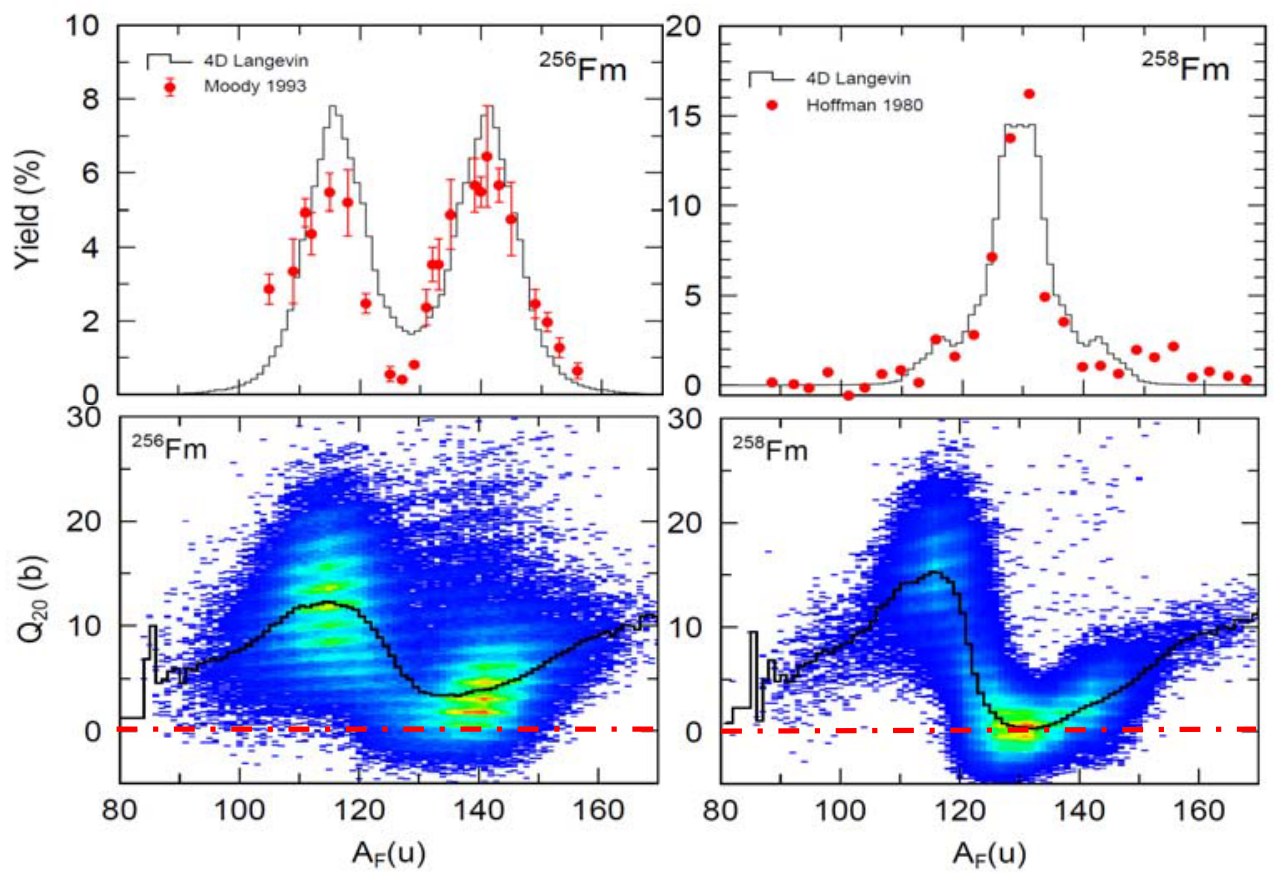

Figure 3 shows mass distributions (upper panels) and mass-quadrupole moment (bottom panels) for ${ }^{256} \mathrm{Fm}$ and ${ }^{258} \mathrm{Fm}$. 


\section{References}

1. M.D.Usang, F.A.Ivanyuk, C.Ishizuka and S.Chiba, Scientific Reports 9, 1525(2019)

2. Y.Abe, S.Ayik, P.-G.Reinhard and E. Suraud, Phys. Rep. 275, 49-196 (1996).

3. J.Maruhn and W.Greiner, Zeit. f. Phys. 251, 431-457 (1972).

4. F.A.Ivanyuk, C.Ishizuka, M.D.Usang and S.Chiba, Phys. Rev. C 97, 054331 (2018).

5. V.V.Pashkevich, Nucl. Phys. A 169, 275-293 (1971).

6. V.V.Pashkevich, Nucl. Phys. A 477, 1-17 (1988).

7. K.T.R.Davies, A.J.Sierk and R.Nix, Phys. Rev. C 13, 2385-2403 (1976).

8. J.Blocki et al Ann. Phys. 113, 330-381 (1978).

9. A.J.Sierk and J.R.Nix, Phys. Rev. C 21, 982-987 (1980).

10. H.Hofmann and D.Kiderlen, Int. Jour. Mod. Phys. E 7, 243-274 (1998).

11. M.D.Usang, F.A.Ivanyuk, C.Ishizuka and S.Chiba, Phys. Rev. C 94, 044602 (2016).

12. C.Ishizuka, M.D.Usang, F.A.Ivanyuk, J.A.Maruhn, K.Nishio and S.Chiba, Phys. Rev. C 96, 064616 (2017).

13. J.P.Unik, and J.E.Gindler, "Critical review of the energy released in nuclear fission", ANL-7748. Tech. Rep., Argonne National Lab., Ill.(1971).

14. V.E.Viola, K.Kwiatkowski, and M.Walker, Phys. Rev. C 31, 1550 (1985).

15. U.Brosa, S.Grossmann and A.Mueller, Phys. Rep. 197, 167-262(1990)

16. G.Scamps and C.Simenel, Nature 546, 382-385(2018) 